\title{
Adesão tardia e as representações sociais relacionadas à assistência pré-natal
}

\author{
Lise Maria Carvalho Mendes (iD \\ Escola de Enfermagem de Ribeirão Preto - Universidade de São Paulo - Ribeirão Preto (SP) - Brasil \\ Graciano Almeida Sudré iD \\ Escola de Enfermagem de Ribeirão Preto - Universidade de São Paulo - Ribeirão Preto (SP) - Brasil \\ Jordânia Vieira Oliveira (iD \\ Universidade Federal do Amapá - Macapá (AP) - Brasil \\ Nayara Gonçalves Barbosa (iD \\ Escola de Enfermagem de Ribeirão Preto - Universidade de São Paulo - Ribeirão Preto (SP) - Brasil \\ Juliana Cristina dos Santos Monteiro iD \\ Escola de Enfermagem de Ribeirão Preto - Universidade de São Paulo - Ribeirão Preto (SP) - Brasil \\ Flavia Azevedo Gomes-Sponholz iD \\ Escola de Enfermagem de Ribeirão Preto - Universidade de São Paulo - Ribeirão Preto (SP) - Brasil
}

\section{RESUMO}

Objetivo: Compreender a adesão tardia e as representações sociais relacionadas à assistência pré-natal. Métodos: Pesquisa descritiva, com abordagem qualitativa para a qual se adotou como referencial metodológico a Análise de Conteúdo, modalidade temática, e como referencial teórico a Teoria das Representações Sociais, que possibilitou interpretar a realidade vivenciada pelo grupo social investigado, para enterder como elaboraram a gama de conhecimento que direcionaram suas ações. Resultados: Por meio do referencial metodológico da Análise de Conteúdo, modalidade temática, emergiram duas categorias: interpretação social da assistência pré-natal e; situações que interferem na qualidade do cuidado pré-natal. As ideias centrais de cada categoria foram interpretadas com apoio da Teoria das Representações Sociais, que permitiu interpretar as ideias centrais sobre pré-natal e as representações dos diferentes discursos. Conclusão: As representações sociais das puérperas sobre pré-natal remetem aos sentidos de prevenção, de acolhimento e de aprendizagem, possivelmente pelo fato de terem aderido tardiamente ao pré-natal, razão pela qual atribuem diferentes obstáculos à adesão, sobretudo relacionados a aspectos socioculturais uma vez que estão imersas no cotidiano fronteiriço amazônico.

Descritores: Cuidado Pré-Natal; Saúde na Fronteira; Gravidez.

\section{INTRODUÇÃO}

O acesso a um pré-natal que propicie cuidados qualificados durante a gravidez é crucial para a redução da mortalidade materna, sobretudo em países caracterizados por altos níveis de pobreza e que não dispõem de um sistema de saúde financiado pelo Estado(1).

No contexto brasileiro, os serviços pré-natais estão presentes no escopo das ações realizadas pelo Sistema Único de Saúde (SUS). No entanto, mesmo em regiões com alta cobertura da atenção pré-natal, a qualidade do cuidado é um processo mais complexo, que demanda investimentos governamentais, para oferta de ações essenciais que condizem com as necessidades de saúde apresentadas. Entretanto, no Brasil, constatam-se ainda disparidades no acesso que são determinadas por fatores sociais e geográficos ${ }^{(2)}$, como ocorre, por exemplo, com os povos da floresta, em que se verifica dificuldade à adesão da gestante ao pré-natal, baixo número de consultas realizadas por essa população e índices elevados de morbidade e de mortalidade materna ${ }^{(3)}$. Esse fato é potencialmente agravado ao considerar que, em relação às orientações às gestantes durante o pré-natal, mesmo diante do acesso,

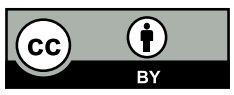

Este artigo está publicado em acesso aberto (Open Access) sob a licença Creative Commons, que permite uso, distribuição e reprodução em qualquer meio, sem restrições, desde que o trabalho seja corretamente citado. 
existem algumas lacunas, como a baixa prevalência da oferta de informações quanto aos sinais de risco, que pouco contribuem para o período gravídico e puerperal bem-sucedidos ${ }^{(4)}$.

Neste processo de reconhecimento da existência de diferentes prejuízos à assistencia pré-natal e não alcance a alguns princípios doutrinários do SUS, como equidade, integralidade e universalidade, foi possível verificar na literatura, a existência de uma assistência insatisfatória em algumas localidades ${ }^{(2,5,6)}$. Problemas decorrentes de inadequação dos recursos físicos, ambientais, humanos, materiais e da fragilidade de sistemas de articulação da rede de atenção à saúde(5).

Toda essa problemática presente no contexto brasileiro suscitou o interesse para o desenvolvimento de um estudo que possibilitasse o entendimento que as mulheres que aderiram tardiamente ao pré-natal têm sobre as representações sociais relacionadas à temática, em especial do extremo norte do país, em um processo de articulação dos achados com a Teoria das Representações Sociais (TRS) ${ }^{(7)}$.

Diante dessas inquietações o presente estudo objetivou compreender a adesão tardia e as representações sociais relacionadas à assistência pré-natal. Ao considerar o processo de subjetivação para alcance dessa possibilidade interpretativa, a relevância desse estudo assenta-se no desvelar das representações de gestantes advindas de um contexto social que pouco favorece a busca pelo cuidado, mesmo que de forma tardia. Sua importância também se dá pela possibilidade de demarcar as representações que emergem ao se depararem com um sistema que produz um cuidado, por vezes insuficiente, situação que enseja reflexões importantes e estimuladoras de ações em saúde mais adequadas e abrangentes, produtoras de mudança.

\section{MÉTODOS}

Pesquisa descritiva, com abordagem qualitativa ${ }^{(8)}$ que adotou a TRS como referencial para interpretação e compreensão dos resultados $^{(7)}$, visando interpretar a realidade vivenciada pelo grupo social investigado, de maneira a enterder como elaboraram a gama de conhecimentos que direcionaram suas ações.

Em relação ao local de estudo, essa pesquisa foi desenvolvida em um município do extremo norte do Brasil, em região de fronteira com a Guiana Francesa, com população de 24 mil habitantes, dentre os quais oito mil indígenas. $O$ acesso à região é prejudicado pelas condições da rodovia que conduz à capital do estado, situada a $590 \mathrm{~km}$ de distância.

O serviço de saúde desse município eleito para a construção do estudo foi um hospital com gestão estadual, de média complexidade, referência regional para obstetrícia, dentre outras especialidades. As participantes da pesquisa foram mulheres que estavam no alojamento conjunto e que obedeciam aos seguintes critérios de inclusão: brasileiras, puerpério imediato, residentes em situação de fronteira e que realizaram pré-natal com início tardio (iniciadas após a décima segunda semana de gestação). Por sua vez, os critérios de exclusão se limitaram às puérperas que apresentavam alguma condição neuropsíquica e àquelas com intercorrências durante o trabalho de parto ou no pós-parto.

Atendendo a esses critérios, 13 mulheres aceitaram participar da pesquisa, em um total de 15 que atendiam a todos os critérios. Dentre os motivos de recusa destacaram-se melancolia, angústia e sentimento de impotência em relação ao trabalho de parto. Para coleta de dados foi utilizada a técnica de entrevista semiestruturada ${ }^{(8)}$; a ordem das entrevistas obedeceu à mesma ordem de admissão na sala de pós-parto imediato e, para cessar a busca por novas respondentes, adotou-se o critério de saturação dos dados ${ }^{(9)}$. A coleta de dados ocorreu durante o mês de novembro de 2017.

O entrevistador possuía formação de nível superior (enfermagem), com experiência em coleta de dados para pesquisas qualitativas. $\mathrm{O}$ ambiente escolhido possiblitava a ausência de interrupções e os temas que guiaram as perguntas basicamente permeavam a percepção sobre o pré-natal acerca dos temas: o atendimento e a disposição profissional e pessoal durante as consultas, bem como a procura tardia e o engajamento pessoal e profissional.

Como ferramenta utilizou-se gravador de áudio que possibilitou a gravação de todas as entrevistas que foram transcritas na íntegra e submetidas à validação de conteúdo pelas respondentes. O tempo médio de duração foi de aproximadamente 30 minutos e para a análise dos dados utilizou-se a Análise de Conteúdo ${ }^{(10)}$, modalidade temática.

A etapa de análise foi realizada por um grupo de pesquisa interprofissional com formação em ciencias da saúde e domínio do processo de análise de dados em entrevistas qualitativas. O processo era composto então por três etapas: pré-análise com organização das unidades de registro e de contexto; exploração do material para reordenação dos dados e estabelecimento de esquemas de classificação e; exploração do material. O conteúdo foi organizado por tendências interpretativas presentes no fenômeno investigado e surgimento de pontos de convergências para codificação e categorização(11). 
Para que a análise fosse guiada pela TRS, a aproximação, o estudo e a compreensão das condições sociais, bem como o entendimento do contexto histórico e das características das mulheres daquela região foram imprescindíveis uma vez que essa estratégia possibilitou maior clareza e assertividade no processo de desvelação das representações sociais. Além disso, o processo interpretativo foi demarcado pelos conceitos de objetivação e de ancoragem: o primeiro materializando o conteúdo apresentado e; o segundo transformando esse conteúdo em algo próximo, passível de elucidação e de distribuição de sistemas particulares de categorias ${ }^{(7)}$.

Visando à matutenção do anonimato das participantes, durante o processo de codificação foi atribuído para cada puérpera a letra $\mathrm{P}$ (letra inicial da palavra puérpera) e um número romano, do I ao XIII, atribuído à medida que as entrevistas ocorriam.

Essa pesquisa foi aprovada pelo Comitê de Ética em Pesquisa (CEP) e cumpriu todas as exigências dispostas na Resolução $n^{\circ} 466 / 2012$, sobretudo no que se refere a solicitar assinatura de cada participante no Termo de Consentimento Livre e Esclarecido (TCLE), para posteriormente proceder à coleta de dados. Após apreciação do CEP, a pesquisa foi aprovada sob Parecer $n^{\circ} 2.155 .712$.

\section{RESULTADOS E DISCUSSÃO}

Por meio do referencial metodológico da Análise de Conteúdo, modalidade temática, emergiram duas categorias: interpretação social da assistência pré-natal e; situações que interferem na qualidade do cuidado pré-natal.

As ideias centrais de cada categoria foram interpretadas com apoio da Teoria das Representações Sociais, que permitiu interpretar as ideias centrais sobre pré-natal e as representações dos diferentes discursos.

Na primeira categoria, interpretação social da assistencia pré-natal, o primeiro processo de objetivação formulou a imagem de "corpos saudáveis", por meio de projeções ancoradas nas ações de cuidado que estavam presentes durante a consulta de modo a mapear a saúde do bebê e acompanhar a saúde da mulher durante o período gestacional:

"Saber como é que está o bebê, ter cuidado para não correr nem um tipo de risco para saber como vai correr todos os meses, ou algum risco de alguma coisa. Para me prevenir, prevenir o bebê." (PI)

"Pré-natal é importante por causa da saúde do bebê e a minha também! Porque se eu tiver com alguma doença, pode passar para o bebê!" (PV)

Outra imagem presente que ocupou o eixo central nessa categoria, pelo fato de traduzir alguns tópicos que remetessem à boa vinculação e ao seguimento do pré-natal, foi a do "abraço". Não na acepção literal do abraço físico, mas na significação relacionada ao pré-natal como um momento de acolhimento, algo necessário para resolução dos problemas durante a gravidez, ao propiciar o surgimento de sentimentos de segurança e de cuidado:

"O pré-natal foi muito importante, me ajudou bastante no momento que eu estava precisando, porque tive um problema na gravidez, eu ia pro posto, eles me tratavam muito bem, me acolhiam, sei que me deram muita segurança lá." (PIX)

"Já é o quarto filho! E eu tive esses problemas de pressão, aí você sabe que tem que cuidar." (PIV)

"Meu primeiro filho nasceu prematuro. Com isso, eu vim, tem que evitar novamente outro parto prematuro." (PVIII)

Em última análise, nessa primeira categoria, objetivada pela figura do "corpo que aprende" a cuidar, o conceito de pré-natal estava ancorado no processo de aprendizagem, propiciado pelo compartilhamento de saberes e pelas decobertas decorrentes da aquisição de novos conhecimentos:

"Com o pré-natal, descobri várias coisas que eu não sabia. O que a gente deve fazer com os bebês." (PX)

"Eu acho importante! Porque nós sabemos o que pode comer para não sentir enjoo e para tomar os remédios de forma correta." (PVII)

As imagens que surgiram - corpos saudáveis, abraço, corpo que aprende - descrevem de forma singular as intenções do pré-natal por um viés que considera o desenvolvimento de habilidades que consideram o manejo da gestação, a afetividade e os aspectos cognitivos. Dessa forma, desvela-se o conhecimento das puérperas em relação ao período vivenciado, como algo de intenso crescimento pessoal que envolve situações de aprendizagem para o cuidado do seu corpo e do corpo do bebê, numa relação de apoio.

A segunda categoria, situações que interferem na qualidade do cuidado pré-natal, possiblitou desvelar, pela identificação dos processos de objetivação e ancoragem, os obstáculos que surgiram nas situações vivenciadas que ditavam sentimentos de ruptura, de descontinuidade e de adesão aos cuidados pré-natais. 
Prevenir os agravos com concepto e recém-nascido durante o período da gestação, do parto e do puerpério, constituíram representações permeadas pelo imaginário das intercorrências que surgem, principalmente entre as multíparas com antecedentes de complicações obstétricas.

Outros estudos também observaram a atitude de prevenção significando e ditando a assistência pré-natal, em especial quando relacionada ao crescimento e ao desenvolvimento saudáveis. Essa apreensão pode estar associada aos elevados índices de morbimortalidade durante o período neonatal precoce, na região, que é superior à média nacional(12).

Como espaço de aprendizagem, é possível observar na literatura que mulheres de baixa renda, assim como mulheres residentes da região Norte, sobretudo residentes em municípios de pequeno porte, recebem menos orientações e ações educativas adequadas durante o pré-natal ${ }^{(6)}$. Todavia, as representações das participantes deste estudo podem ser apontadas como um reflexo positivo sobre a qualidade da assistência, uma vez que as participantes observaram no pré-natal um espaço para a discussão de suas dúvidas.

Inclinando-se a uma interpretação diante das necessidades relacionais, um dos eixos propostos permeia o aspecto do acolhimento, seja por meio da assistência propriamente dita ou pelo encontro e troca de saberes. $O$ acolhimento é parte estruturante e agente determinante para o sucesso da assistência pré-natal, uma vez que fortalece a relação dos profissionais de saúde com a comunidade e em seus diversos contextos de atuação(13,14).

O acolhimento, que esteve ancorado nos discursos das puérperas, foi percebido como uma das motivações para a adesão e para a continuidade das consultas de pré-natal, oportunidade em que as puérperas relataram a importância do vínculo para a formulação de um sentimento de segurança e de autoconfiança.

Surgiu como importante estratégia do pré-natal a ação de disponibilizar um canal de diálogo, como estratégia de atenção à saúde das gestantes, oportunizando assim um local acessível, instituído nas relações de cuidado entre trabalhador da saúde e mulher em seu período gravídico-puerperal, com atribuição de valores que emergem do encontro, emergindo limitações impregnadas pelo contexto sociocultural.

O primeiro processo de objetivação nesse bloco de análise permitiu a construção da imagem de "solidão", ancorada pela ausência de redes de apoio durante o seguimento do pré-natal:

"Eu não fui mais fazer [o pré-natal] porque tenho três meninos, daí, você sabe, é difícil ter com quem deixar. O pai deles, meu marido, está lá no barranco [garimpo]. Aqui não tenho ninguém, nem uma irmã pra ficar olhando eles, não dá pra descer o rio com eles sempre." (PIV)

"Eu ainda estava na escola, não tinha com quem contar; minha mãe não aceitou eu estar grávida de jeito nenhum. Ai eu e o pai dele tivemos que trabalhar e não tive como ir no posto." (PVII)

Quando as necessidades de saúde convencionais - além daquelas pautadas no autocuidado e no autogoverno - demandavam um apoio adicional, elas indicavam para um processo de ruptura como a saída mais viável.

A outra imagem que emergiu foi a de "pote vazio", aconrada nos relatos que justificavam as rupturas pela desorganização dos serviços ou não correspondência às necessidades pessoais, refletidas por determinações sociais de uma realidade que determina os processos de seguir a vida de outras formas, despertando sentimentos de abandono por parte do Estado:

"A falta de dinheiro para realizar exames particulares que não tem aqui." (PVIII)

"Eu sou do Cassiporé, lá não tem exame pra nada, pra eu vir pra cidade gasto dinheiro aqui e não resolvo nada. Não tem como estar todo mês aqui [sede urbana], não!" (PII)

"É ruim porque não tem os remédios que a doutora passa, aí a gente fica de um lado pro outro e chega a ter o menino e não recebe nada". (PI)

Finalizando a estrutura interpretativa nessa categoria, surge a imagem do "corpo destinado", situação ancorada pelos aspectos socioculturais:

"Meu marido é pirateiro e eu sou marreteira [comerciante móvel], não é? Aí eu trazia umas coisas de Goiânia e de Fortaleza pra vender aqui. Às vezes eu estava lá em Macapá, às vezes no mundo [risos], às vezes eu estava aqui. Não tive como fazer pré-natal direitinho não, mas aí nasceu prematuro [choro]." (PVIII)

"Ela só fez uma consulta aqui na cidade, com a enfermeira; lá na aldeia só tinha médico e o marido dela não deixava ela ir pra consultas porque ele era homem. Tem também a nossa língua, né? Só os homens e as velhas que falam português." (PXI) 
A adesão ao cuidado pré-natal nesse caso relacionava-se ao contexto onde a puérpera encontrava-se durante sua gestação, caracterizado pelo não acompanhamento, situação que coloca a atenção pré-natal em segundo plano devido à importância atribuída às demais situações vivenciadas.

Nessa segunda categoria estão demarcadas algumas representações pautadas na ausência de redes de apoio social. A partir do suporte emocional e psíquico de pessoas próximas à gestante, a mulher sente-se mais segura e acolhida. Nessa perspectiva, o apoio de familiares, de amigos, de vizinhos e de profissionais de saúde é imprescindível à adesão das consultas pré-natais, podendo configurar-se como um fator determinante para adesão e manutenção da assistência ${ }^{(14)}$.

O apoio social é uma ferramenta de suporte psicológico e emocional à maternidade, capaz de gerar condições satisfatórias e facilitadoras de interação entre os novos pais e o bebê, propiciando assim o exercício das novas funções como cuidadores. Nesse sentido, esse apoio torna-se de extrema importância para o enfrentamento de situações estressantes, além de possibilitar a adequação de comportamentos maternos em relação aos filhos ${ }^{(13)}$.

A distância para conseguir o atendimento de que precisam, a demora para o agendamento de consultas e de exames, a restrição para aquisição de medicamentos e a falta de dinheiro que inviabiliza o pagamento por serviços não ofertados pelos SUS representaram obstáculos à adesão precoce e ao seguimento das consultas.

Alguns municípios tornam-se referência para povos indígena, tendo em vista o oferecimento de serviços definidos pela política de atenção à saúde indígena pela Casa de Saúde Indígena (CASAI), que oferece apoio às gestantes indígenas que precisam de assistência de saúde. Algumas gestantes indígenas realizam a assistência pré-natal nas unidades de saúde em suas aldeias, utilizando a CASAl como espaço de apoio para fixação e por vezes moradia, apenas quando necessitam de assistência especializada ou hospitalar ${ }^{(14)}$.

Inúmeras gestantes moram em áreas rurais de difícil acesso, onde não há serviços de saúde próximos, a exemplo de áreas de garimpos e assentamentos. Estudos apontam que uma situação socioeconômica desfavorável e o difícil acesso demográfico, como as verificadas neste estudo, dificultam o acesso das gestantes às consultas pré-natais ${ }^{(15)}$.

A distância física e o tempo de viagem são classificados como barreiras relacionadas ao acesso a esses serviços. Corroborando estes relatos, verifica-se que a situação de isolamento geográfico ainda afeta a atenção à saúde da mulher na Amazônia, posto que a localização da residência se mostrou significativamente associada ao atendimento pré-natal no primeiro trimestre, com menor acesso às mulheres provenientes da zona rural ${ }^{(16)}$.

Considerando que o SUS orienta a universalidade e a integralidade na oferta de serviços, os custos diretos à realização da assistência pré-natal deve ser considerados para análise e reorganização da estrutura dos serviços em lugares de difícil acesso ${ }^{(14)}$. Esses custos atuam aumentando as desigualdades sociais no acesso à assistência de saúde, em que gestantes mais pobres são mais atingidas ${ }^{(17)}$.

As condições de trabalho presentes no contexto de fronteira amazônica, principalmente as que mobilizam um grande fluxo nessa região, como o garimpo, são exemplos de aspectos socioculturais associados às representações de obstáculos à adesão ao pré-natal.

Importante destacar nesse contexto que a garimpagem é uma das principais fontes de recursos financeiros da região onde a pesquisa foi realizada. Na maioria das vezes, o acesso aos garimpos leva cerca de 70 horas de viagem da sede urbana, dificultando assim a adesão às consultas de pré-natal por parte das mulheres que trabalham nessa atividade, constituindo uma das inúmeras barreiras físicas e financeiras ${ }^{(14)}$.

Dessa maneira, a gestante garimpeira precisa ausentar-se de sua atividade laboral, perdendo sua única fonte de renda. Não obstante, essas mulheres, muitas vezes, encontram-se em condições de irregularidade, trabalhando em garimpos clandestinos situados em áreas de floresta na Guiana Francesa, sem documentos de registo civil, o que as impedem de receber auxílios de transferência de renda do governo brasileiro.

Outro exemplo da influência sociocultural está presente na região fronteiriça de Oiapoque-Tumucumaque, composta por inúmeras etnias indígenas e outros povos da floresta. Idosos e homens são intérpretes da gestante, uma vez que a mulher, da aldeia Kumenê, da etnia Palikur, por exemplo, não compreendem o idioma português, somente o parikwaki, filiada à grande família linguística Arawak.

Institui-se nesse processo uma barreira à comunicação entre a gestante e o profissional, situação essa que pode representar uma adesão tardia e/ou descontinuidade das consultas ${ }^{(14)}$. $O$ dialeto torna-se um agente limitante à compreensão real do que a paciente refere sobre sua saúde, por mais que haja intérpretes ${ }^{(15)}$.

Entre algumas mulheres indígenas foi observado que a realização do pré-natal está condicionada à autorização do pai da criança. A narrativa elaborada por algumas mulheres denotam que o percurso e o acesso aos serviços são vivenciados de modo distinto por cada uma e assim os contornos de uma assistência pré-natal podem estar assinalados por questões diferentes entre si, inclusive pelo fato de ser mulher. 


\section{CONSIDERAÇÕES FINAIS}

As representações sociais das puérperas investigadas sobre pré-natal remetem ao sentido de prevenção, de acolhimento e de aprendizagem. Em virtude da adesão tardia ao pré-natal atribuem diferentes obstáculos à adesão, com destaque para aqueles relacionados a aspectos socioculturais, tendo em vista a realidade cotidiana da região fronteiriça amazônica na qual estão inseridas.

Diante desse cenário, surgem distintas conotações ao pré-natal, geralmente vinculadas: às precárias relações trabalhistas; à violência de gênero; às variabilidades linguística e cultural; à ausência das redes de apoio e; à desorganização dos serviços que se encontram distantes, com barreiras no acesso ditadas pela morosidade do sistema de agendamento e de regulação.

Conclui-se que as representações apontadas podem direcionar pesquisas futuras acerca da adesão ao prénatal, com destaque para o aprofundamento da temática do acompanhamento de gestantes, problematizando as relações trabalhistas precárias e a necessidade de capacitação dos profissionais de saúde para assistência no contexto de diversidades étnicas e culturais.

Embora o tipo de estudo adotado para o desenvolvimento da presente investigação impossibilite a generalização dos dados, os resultados contribuíram de forma significativa para o desvelar dos cuidados pré-natais na região estudada, permitindo compreender as particularidades vivenciadas por algumas mulheres e os significados que permeavam o processo de busca pela saúde no período gestacional.

As contribuições desta pesquisa qualitativa inserem-se outrossim no conhecimento das representações sociais que impactam diretamente na adesão à consulta do pré-natal entre povos da floresta. Estudos dessa natureza são essenciais para trazer à tona os fenômenos vivenciados por sujeitos que experenciam a atenção pré-natal em regiões consideradas vulneráveis, favorecendo a avaliação e o direcionamento de políticas públicas nacionais e internacionais.

\section{CONFLITOS DE INTERESSE}

Os(As) autores(as) declaram não haver conflitos de interesses.

\section{CONTRIBUIÇÕES}

Todos os autores contribuíram com a elaboração e delineamento do estudo; análise e interpretação de dados e; redação e revisão do manuscrito. Todos aprovaram a versão final enviada para publicação na Revista Brasileira em Promoção da Saúde (RBPS) e são responsáveis por todos os aspectos do trabalho.

\section{AGRADECIMENTOS}

Autores agradecem ao CIAIQ, CAPES e CNPq.

\section{FONTES DE FINANCIAMENTO}

Coordenação de Aperfeiçoamento de Pessoal de Nível Superior - Brasil (CAPES) - Código de Financiamento 001.

\section{REFERÊNCIAS}

1. Udenigwe O, Okonofua FE, Ntoimo LFC, Imongan W, Igboin B, Yaya S. Perspectives of policymakers and health providers on barriers and facilitators to skilled pregnancy care: findings from a qualitative study in rural Nigeria. BMC Pregnancy and Childbirth. 2021;21(1):20.

2. Esposti CDD, Santos-Neto ET dos, Oliveira AE, Travassos C, Pinheiro RS. Desigualdades sociais e geográficas no desempenho da assistência pré-natal de uma Região Metropolitana do Brasil. Ciência \& Saúde Coletiva. 2020;25(5):1735-50.

3. Tomasi E, Fernandes PAA, Fischer T, Siqueira FCV, Silveira DS da, Thumé E, et al. Qualidade da atenção pré-natal na rede básica de saúde do Brasil: indicadores e desigualdades sociais. Cadernos de Saúde Pública. 2017;33(3). 
4. Marques BL, Tomasi YT, Saraiva S dos S, Boing AF, Geremia DS. Orientações às gestantes no pré-natal: a importância do cuidado compartilhado na atenção primária em saúde. Escola Anna Nery. 2021;25(1).

5. Nascimento LC dos S, Silva MRF da, Abreu PD de, Araújo EC de, Menezes MLN de, Oliveira ECT. Perspectiva dos enfermeiros sobre a assistência pré-natal no âmbito da Estratégia Saúde da Família. Revista de Enfermagem da UFSM. 2020:16(10):e44.

6. Tomasi E, Fernandes PAA, Fischer T, Siqueira FCV, Silveira DS da, Thumé E, et al. Qualidade da atenção pré-natal na rede básica de saúde do Brasil: indicadores e desigualdades sociais. Cadernos de Saúde Pública. 2017;33(3).

7. Moscovici S. Representações sociais: investigação em psicologia social. Petrópolis, RJ: Vozes; 2003.

8. Minayo MC de S, Costa AP. Fundamentos teóricos das técnicas de investigação qualitativa. Revista Lusófona de Educação [Internet]. 2018 [cited 2021 Jul 12];40:139-53. Available from: https://ria.ua.pt/ handle/10773/26788

9. Minayo MC de S. Amostragem e saturação em pesquisa qualitativa: consensos e controvérsias. Revista Pesquisa Qualitativa [Internet]. 2017 [cited 2020 May 15];5(7):1-12. Available from: https://editora.sepq.org.br/ index.php/rpq/article/view/82

10. Bardin L. Análise de Conteúdo. São Paulo: Edições 70; 2016. 279.

11. Minayo MC de S, Deslandes SF, Gomes R. Pesquisa Social: teoria, método e criatividade. Série Manu. Petrópolis, RJ: Vozes; 2019.

12. Gama ASM, Fernandes TG, Parente RCP, Secoli SR. Inquérito de saúde em comunidades ribeirinhas do Amazonas, Brasil. Cadernos de Saúde Pública [Internet]. 2018 [cited 2021 Aug 7];34(2). Available from: http:// www.scielo.br/j/csp/a/nWyTKM4WRV5Gxr4pSVT4Mnp/abstract/?lang=pt

13. Gonçalves ITJP, Souza KV, Amaral MA, Oliveira ARS de, Ferreira WFC. Prática do acolhimento na assistência pré-natal: limites, potencialidades e contribuições da enfermagem. Rev Rene [Internet]. 2013 [cited 2021 Aug 7];14(3). Available from: http://www.periodicos.ufc.br/rene/article/view/3503

14. Mendes LMC, Sudré GA, Oliveira JV, Barbosa NG, Monteiro JC dos S, Gomes-Sponholz FA. Representações sociais de puérperas sobre a adesão ao pré-natal na fronteira franco-brasileira. New Trends in Qualitative Research. 2021;8:130-7.

15. Silva AC, Mendes LMC, Monteiro RS, Silva RA, Pinheiro AKB. Parto e nascimento na fronteira francobrasileira: percepções de enfermeiros. Cogitare Enfermagem. 2020 Jun 11;25.

16. Moore N, Blouin B, Razuri H, Casapia M, Gyorkos TW. Determinants of first trimester attendance at antenatal care clinics in the Amazon region of Peru: A case-control study. Montazeri A, editor. PLOS ONE [Internet]. 2017 [cited 2021 Aug 7];16;12(2):e0171136. Available from: https://dx.plos.org/10.1371/journal.pone.0171136

17. Esposti CDD, Oliveira AE, Santos ET dos, Travassos C. Representações sociais sobre o acesso e o cuidado pré-natal no Sistema Único de Saúde da Região Metropolitana da Grande Vitória, Espírito Santo1. Saúde e Sociedade [Internet]. 2015 [cited 2021 Aug 7];24(3):765-79. Available from: http://www.scielo.br/j/sausoc/a/ zHDcSThr6GhsVkdJxhW6L5m/abstract/?lang=pt

\section{Endereço da Primeira Autora:}

Lise Maria Carvalho Mendes

Escola de Enfermagem de Ribeirão Preto - Universidade de São Paulo

Avenida dos Bandeirantes, 3900

Campus Universitário - Bairro Monte Alegre

CEP: 14040-902 - Ribeirão Preto - SP - Brasil

E-mail: lisedemendes@usp.br

\section{Endereço para correspondência:}

Graciano Almeida Sudré

Escola de Enfermagem de Ribeirão Preto - Universidade de São Paulo

Avenida dos Bandeirantes, 3900

Campus Universitário - Bairro Monte Alegre

CEP: 14040-902 - Ribeirão Preto - SP - Brasil

E-mail: gracianosudre@usp.br 